\title{
Ticagrelor versus Prasugrel in Patients with Acute Coronary Syndrome Undergoing Percutaneous Coronary Intervention: Analysis from the Acute Coronary Syndrome Israeli Survey
}

\author{
Ran Eliaz ${ }^{\mathrm{a}}$ Bethlehem Mengesha ${ }^{\mathrm{b}, \mathrm{c}}$ Tal Ovdat ${ }^{\mathrm{d}}$ Zaza lakobishvili $^{c, \mathrm{e}}$ \\ David Hasdai ${ }^{c, f}$ Mark Kheifets ${ }^{c, f}$ Robert Klempfner ${ }^{c, d, g}$ Roy Beigel ${ }^{c, g}$ \\ Eran Kalmanovich ${ }^{b, c}$ Ronny Alcalai ${ }^{a}$ Amos Levi ${ }^{c, f}$ \\ aDepartment of Cardiology, Hadassah Medical Center, Israel and the Hebrew University of Jerusalem, Faculty of \\ Medicine, Jerusalem, Israel; bShamir Medical Center, Be'er Ya'acov, Israel; 'The Sackler Faculty of Medicine, Tel Aviv \\ University, Tel Aviv, Israel; 'Israel Center for Cardiovascular Research, Sheba Medical Center, Ramat-Gan, Israel;

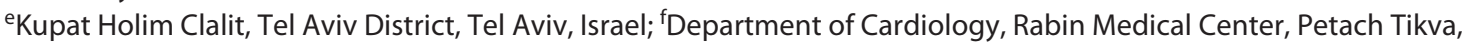 \\ Israel; ${ }^{9}$ The Cardiovascular Division Sheba Medical Center, Department of Cardiology, Tel-Hashomer, Ramat-Gan, Israel
}

\section{Keywords}

Percutaneous coronary intervention - Prasugrel · Ticagrelor . Acute coronary syndrome

\begin{abstract}
Introduction: We aimed to compare the outcomes of acute coronary syndrome (ACS) patients undergoing in-hospital percutaneous coronary intervention treated with prasugrel versus ticagrelor. Methods: Among 7,233 patients enrolled to the Acute Coronary Syndrome Israeli Survey (ACSIS) between 2010 and 2018, we identified 1,126 eligible patients treated with prasugrel and 817 with ticagrelor. Comparison between the groups was performed separately in ST-elevation myocardial infarction (STEMI) patients, propensity score matched (PSM) STEMI patients, and non-ST-elevation ACS (NSTE-ACS) patients. Results: In-hospital complication rates, including rates of stent thrombosis, were not significantly different between groups. In PSM STEMI patients, 30-day rehospitalization rate $(p<0.05), 30$-day MACE (the composite of death, Ml, stroke, and urgent revascularization, $p=0.006$ ), and 1-year mortality rates $(p=0.08)$ were higher in the ticagrelor group compared to the prasugrel group; in NSTE-
\end{abstract}

karger@karger.com www.karger.com/crd

Karger $\stackrel{\text { ' }}{5}$

BOPEN ACCESS
(C) 2021 The Author(s)

Published by S. Karger AG, Basel

This article is licensed under the Creative Commons Attribution 4.0 International License (CC BY) (http://www.karger.com/Services/ OpenAccessLicense). Usage, derivative works and distribution are permitted provided that proper credit is given to the author and the original publisher.
ACS patients, outcomes were not associated with drug choice. In Cox regression analysis applied on the entire cohort, prasugrel was associated with lower 1-year mortality in STEMI patients but not in NSTE-ACS patients ( $p$ for interaction 0.03). Conclusions: Compared to ticagrelor, prasugrel was associated with superior clinical outcomes in STEMI patients, but not in NSTE-ACS patients.

(c) 2021 The Author(s).

Published by S. Karger AG, Basel

\section{Introduction}

Based on data from randomized controlled trials, both American and European guidelines recommend treating acute coronary syndrome (ACS) patients with secondgeneration P2Y12 inhibitors (i.e., ticagrelor and prasugrel) $[1,2]$. These agents differ both in pharmacological properties and administration protocol. While ticagrelor is administered regardless of coronary angiography, prasugrel administration is recommended only after coro-

Ran Eliaz and Bethlehem Mengesha contributed equally as first authors. Amos Levi and Ronny Alcalai contributed equally as senior authors.
Correspondence to:

Amos Levi, amos.levi@gmail.com 
nary anatomy has been delineated and percutaneous coronary intervention (PCI) is planned, or before angiography in ST-elevation myocardial infarction (STEMI) patients who are treated with primary PCI. This is in keeping with the TRITON-TIMI 38 protocol and the results of the ACCOAST trial, which have demonstrated no benefit for early versus delayed administration of prasugrel in non-ST-elevation ACS (NSTE-ACS) [3, 4]. Direct head-to-head comparison of these agents was scarce until the recent publication of the ISAR-REACT- 5 study which demonstrated the superiority of prasugrel over ticagrelor in terms of 1-year composite of death, myocardial infarction (MI), and stroke [5]. Given the unexpected outcomes of this trial, we aimed to perform a comparison of ticagrelor and prasugrel in real-life ACS patients.

\section{Methods}

The current analysis is an observational study based on data obtained from the Acute Coronary Syndrome in Israel Survey (ACSIS). ACSIS is a national ACS snapshot survey conducted in all 25 cardiology departments in Israel since 2000 over a 2-month period, every $2-3$ years. Data pertaining to all ACS hospitalizations are prospectively collected, using a prespecified electronic case record. The survey is governed and coordinated by the working group on acute cardiovascular care of the Israeli Heart Society, in collaboration with the Israeli Association for Cardiovascular Trials (IACT) and the Israeli Center of Disease Control (ICDC) of the Ministry of Health of the State of Israel. The data storage, maintenance, and processing are performed by the IACT, which also reviews documents to ensure data validity and quality.

Both prasugrel and ticagrelor were commercially introduced in Israel in 2010. We therefore considered patients enrolled in ACSIS 2010-2018 for the present analysis. Data collection for all ACSIS was approved at each hospital by the local institutional ethics review board. All patients were informed of the nature and aims of surveys and signed a written informed consent.

Data on baseline characteristics, risk factors, medical (prior revascularization, MI, heart failure, stroke, peripheral artery disease, and cancer history), and medication history were collected. Information regarding time intervals from the pain onset to the first medical contact and time to reperfusion, transportation data, use of cardiovascular procedures, and pharmacologic management was obtained until discharge. Thirty-day structured telephone follow-up was made by the dedicated study team. Mortality data during hospitalization, at 30 days, and thereafter were determined for all patients from hospital charts and by matching identification numbers of patients with the Israeli National Population Register.

Continuous variables were reported as means $( \pm S D)$, as well medians and interquartile ranges, when appropriate. Categorical variables were described as percentages. Characteristics of study participants were compared using the $\chi^{2}$ test for categorical variables and Student's $t$ test or Wilcoxon rank tests, as appropriate for continuous variables. All tests were 2 -sided, and a value of $p<0.05$ was considered significant. Hazard ratios were accompanied by $95 \%$ confidence intervals. Survival curves were plotted, and the Kaplan-Meier log rank test was used to test the study groups on survival at 1 year. Cox proportional hazards models were used to assess the impact of ticagrelor versus prasugrel on survival, after adjusting for covariates. Covariates were selected based on univariable analysis $(p<0.05)$. To reduce bias between STEMI patients treated with ticagrelor versus prasugrel, a propensity score matching (PSM) was performed. The propensity score evaluates the probability for treating a patient with ticagrelor versus prasugrel using a logistic regression model. Variables which were significantly different between ticagrelor and prasugrel STEMI patients $(p<0.05)$, with $<5 \%$ missing values, or variables which came out statistically significant after matching were included in the model. The following covariates were selected: age, hypertension, current smoker, prior chronic renal failure, and prior MI. PSM was performed with a caliper of 0.01 and a 1:2 matching (ticagrelor:prasugrel), producing groups with 250 ticagrelor patients and 500 prasugrel patients. PSM was not applied in the NSTE-ACS subset given the similarity between the groups in baseline characteristics. Major adverse cardiovascular outcomes (MACE) were defined as the composite of 30-day death, unstable angina pectoris, RE-MI, stroke, stent thrombosis, and urgent revascularization. All analyses were performed using R (R-studio, V.4.0.0; Vienna, Austria).

\section{Results}

Between 2010 and 2018, 7,233 patients were enrolled to the ACSIS. After excluding patients with relative or absolute contraindications to prasugrel (age $\geq 75$ years, weight $<60 \mathrm{~kg}$, and history of prior cerebrovascular event), patients with missing data regarding medical therapy at discharge, and patients who died in the hospital, 4,399 remained (Fig. 1). Of these, 1,100 patients were treated with ticagrelor, 1,234 with prasugrel, and 2,065 with clopidogrel. Although almost all patients were prescribed clopidogrel in 2010, as of 2013, the use of clopidogrel declined to $<10 \%$. Prasugrel was the most common P2Y12 blocker prescribed for ACS patients in 2013, with a gradual shift toward ticagrelor dominance in 2016 and 2018 (Fig. 2). In the current analysis, we excluded the clopidogrel group and all patients who did not undergo in-hospital PCI. Thus, our cohort includes 1,943 patients admitted for ACS who underwent PCI and were initially treated with either prasugrel $(n=1,126)$ or ticagrelor $(n=817)$.

There was a clear correlation between the clinical syndrome - NSTE-ACS versus STEMI - and drug choice: while most STEMI patients were treated with prasugrel $(903 / 1,209$; 74.7\%), most NSTE-ACS were given ticagrelor (511/734; 69.6\%). Given the difference between the syndromes with respect to characteristics and outcomes, we decided to separately analyze NSTE-ACS and STEMI patients.

Baseline and clinical characteristics of STEMI patients treated with either prasugrel or ticagrelor are summarized in Table 1. Patients treated with prasugrel were 


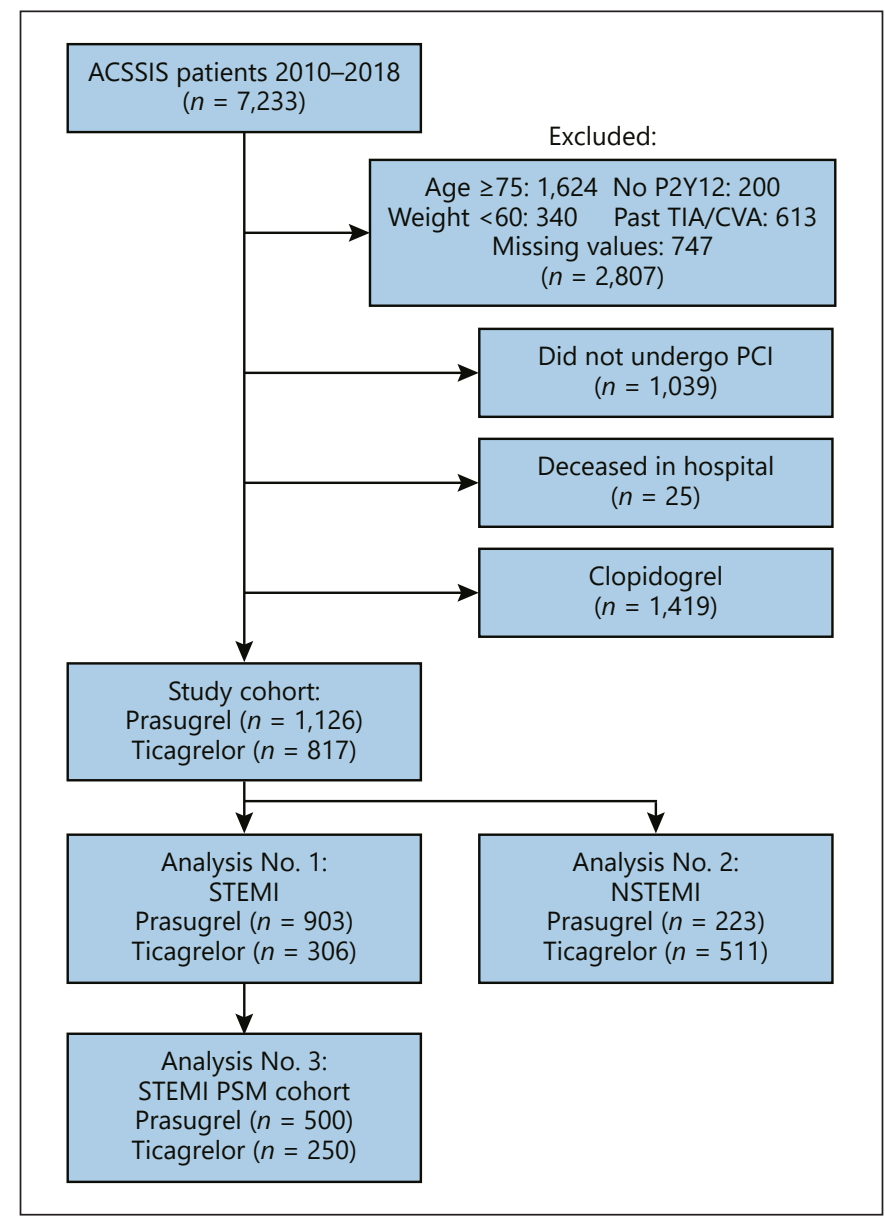

Fig. 1. Study flowchart.

slightly younger and were more commonly smokers. Patients in the ticagrelor group had higher rates of chronic kidney disease (CKD). There were no significant differences between the groups with regard to clinical characteristics on admission. Most patients in both groups were in Killip class I on admission. Most PCIs in both groups (92.3\% in the ticagrelor group and $94.8 \%$ in the prasugrel group) were performed urgently (primary PCI). While peak CPK levels were slightly higher in the prasugrel group, all other laboratory tests were similar in both groups. The rate of in-hospital complications was low and comparable in both groups, except for a higher rate of pulmonary edema $(p=0.016)$ and acute kidney injury $(p=0.026)$ in the ticagrelor group.

Data on the use of medications at 30-day follow-up are summarized in Table 2.

Conversion to clopidogrel occurred more often in the ticagrelor group than in the prasugrel group $(11.7 \%$ vs. $7.7 \%$, respectively, $p=0.06)$. The rate of conversion from

Prasugrel versus Ticagrelor in Acute

Coronary Syndrome

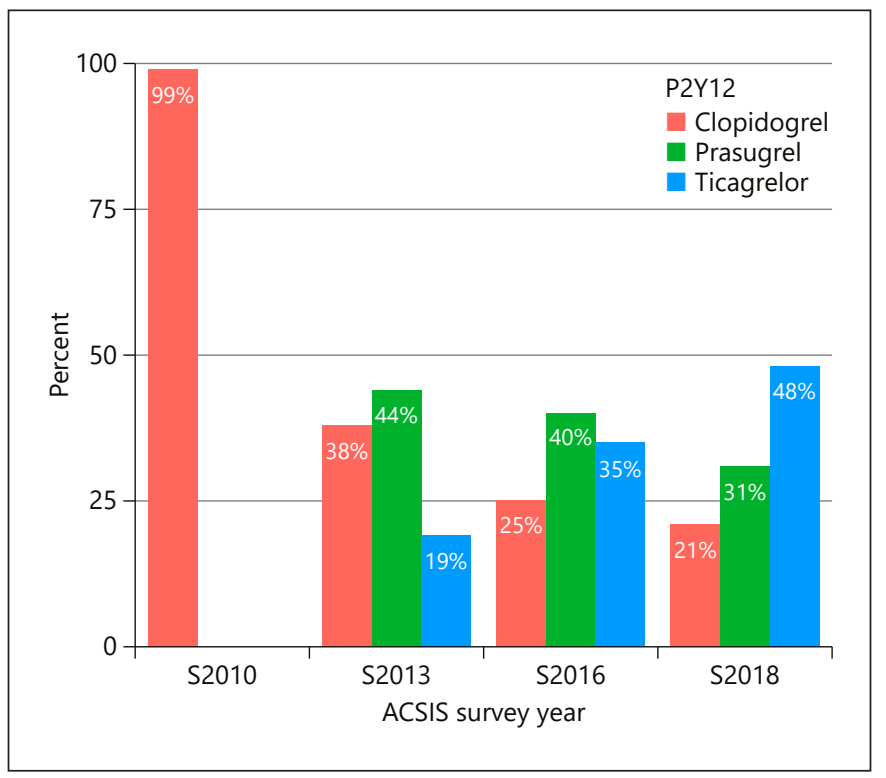

Fig. 2. Temporal trends in P2Y12 inhibitor drug choice.

prasugrel to ticagrelor and vice versa was low for both. Referral to cardiac rehabilitation was more common in the prasugrel group $(p=0.007)$. All other treatments were not significantly different between groups.

Data on 30-day clinical outcomes are shown in Table 3. The rate of re-hospitalizations and overall MACE was significantly higher in the ticagrelor group. Mortality at 30 days was low in both groups but numerically higher in patients treated with ticagrelor compared to prasugrel ( $2 \%$ vs. $0.9 \%, p=0.2)$. One-year mortality was significantly higher in the ticagrelor group ( $4.5 \%$ vs. $2.1 \%$, log rank $=0.033$; Fig. $3 a$ ). In a multivariate Cox model adjusting for age, smoking, and CKD, the hazard ratio for 1-year mortality for patients treated with ticagrelor compared to prasugrel was 1.98 (95\% CI: 0.97-4.07; $p=0.07$ ).

A 2:1 PSM was applied to construct a balanced cohort yielding 250 patients treated with ticagrelor and 500 patients with prasugrel. The baseline characteristics of the study population after matching are shown in Table 1, and the 30-day clinical outcomes are shown in Table 3. The rate of re-hospitalizations and overall MACE was significantly higher in the ticagrelor group. One-year mortality in the ticagrelor group was $4.2 \%$ compared to $1.9 \%$ in the prasugrel group $(\log \operatorname{rank} p=0.08$, Fig. $3 b)$.

Baseline characteristics of NSTE-ACS patients treated with prasugrel and ticagrelor are provided in online supplementary material (for all online suppl. material, see www.karger.com/doi/10.1159/000521042). Patients given prasugrel more often smoked, with otherwise no sig- 
Table 1. Baseline characteristics of STEMI patients

\begin{tabular}{|c|c|c|c|c|c|c|}
\hline & \multicolumn{3}{|l|}{ Entire cohort } & \multicolumn{3}{|l|}{ PSM cohort } \\
\hline & ticagrelor & prasugrel & $p$ value & ticagrelor & prasugrel & $p$ value \\
\hline$N$ & 306 & 903 & & 250 & 500 & \\
\hline \multicolumn{7}{|l|}{ Baseline characteristics, $n(\%)$} \\
\hline Age, years (median [IQR]) & $59.5[52.3,65]$ & $57[50,64]$ & 0.004 & $58[52,65]$ & $59.00[52,65]$ & 0.519 \\
\hline Gender (male) & $275(89.9)$ & $794(87.9)$ & 0.416 & $226(90.4)$ & $433(86.6)$ & 0.166 \\
\hline Dyslipidemia & $184(60.3)$ & $579(64.4)$ & 0.227 & $159(63.6)$ & $323(64.6)$ & 0.850 \\
\hline Hypertension & $162(52.9)$ & $425(47.2)$ & 0.096 & $127(50.8)$ & $251(50.3)$ & 0.959 \\
\hline Current smoker & $150(49.0)$ & $539(59.7)$ & 0.001 & $127(50.8)$ & $245(49.0)$ & 0.699 \\
\hline Diabetes mellitus & $101(33.1)$ & $250(27.7)$ & 0.085 & $80(32.0)$ & $145(29.1)$ & 0.457 \\
\hline Family history of CAD & $103(36.4)$ & $326(40.3)$ & 0.271 & $88(38.1)$ & $184(41.6)$ & 0.421 \\
\hline Prior MI & $72(23.5)$ & $185(20.5)$ & 0.305 & $58(23.2)$ & $97(19.4)$ & 0.270 \\
\hline Prior CABG & $9(2.9)$ & $15(1.7)$ & 0.250 & $7(2.8)$ & $10(2.0)$ & 0.665 \\
\hline Prior $\mathrm{PCl}$ & $71(23.2)$ & $188(20.8)$ & 0.430 & $57(22.8)$ & $99(19.8)$ & 0.398 \\
\hline Chronic renal failure & $16(5.2)$ & $24(2.7)$ & 0.047 & $6(2.4)$ & $7(1.4)$ & 0.489 \\
\hline PVD & $12(3.9)$ & $18(2.0)$ & 0.097 & $3(1.2)$ & $1(0.2)$ & 0.215 \\
\hline History of $\mathrm{CHF}$ & $4(1.3)$ & $19(2.1)$ & 0.522 & $4(1.6)$ & $7(1.4)$ & 1.000 \\
\hline \multicolumn{7}{|l|}{ Prior medical therapy } \\
\hline Aspirin & $87(32.5)$ & $266(31.1)$ & 0.725 & 75 (33.9) & $160(33.4)$ & 0.958 \\
\hline Clopidogrel & $11(4.4)$ & $18(2.2)$ & 0.106 & $9(4.3)$ & $11(2.4)$ & 0.272 \\
\hline ACE-I & $55(23.4)$ & $163(20.3)$ & 0.349 & $44(22.8)$ & $92(20.7)$ & 0.629 \\
\hline ARB & $26(12.7)$ & $69(9.5)$ & 0.239 & $22(12.8)$ & $46(11.3)$ & 0.721 \\
\hline Beta blockers & $52(21.8)$ & $144(18.0)$ & 0.227 & $43(21.4)$ & $89(20.0)$ & 0.774 \\
\hline Statins & $85(44.5)$ & $288(41.1)$ & 0.443 & $71(44.7)$ & $180(45.0)$ & 1.000 \\
\hline Hypoglycemic agents & $58(19.0)$ & $159(17.6)$ & 0.663 & $47(18.8)$ & $96(19.2)$ & 0.974 \\
\hline Diuretics & $11(5.4)$ & $35(4.8)$ & 0.894 & $10(5.8)$ & 20 (4.9) & 0.819 \\
\hline
\end{tabular}

$C A D$, coronary artery disease; CABG, coronary artery bypass grafting; PVD, peripheral vascular disease; $C H F$, congestive heart failure.

Table 2. Drug treatment in STEMI patients, at discharge and at 30-day follow-up

\begin{tabular}{|c|c|c|c|c|c|c|}
\hline & \multicolumn{3}{|c|}{ Entire cohort } & \multicolumn{3}{|c|}{ PSM cohort } \\
\hline & ticagrelor & prasugrel & $p$ value & ticagrelor & prasugrel & $p$ value \\
\hline$N$ & 306 & 903 & & 250 & 500 & \\
\hline \multicolumn{7}{|l|}{ Treatment at discharge } \\
\hline Aspirin & $302(99.0)$ & $886(98.3)$ & 0.565 & $247(99.2)$ & $491(98.4)$ & 0.576 \\
\hline P2Y12-I & $300(98.0)$ & $888(98.3)$ & 0.925 & $245(98.0)$ & $491(98.2)$ & 1.000 \\
\hline Statins & $294(98.3)$ & $865(97.5)$ & 0.558 & $239(98.0)$ & $479(97.6)$ & 0.940 \\
\hline ACE-I/ARB & $261(92.2)$ & $770(90.3)$ & 0.386 & $216(92.3)$ & $441(92.3)$ & 1.000 \\
\hline Beta blockers & $246(87.2)$ & $736(86.8)$ & 0.929 & $204(87.2)$ & $402(85.5)$ & 0.632 \\
\hline \multicolumn{7}{|l|}{ Treatment at 30-day follow-up } \\
\hline Cardiac rehabilitation (referral) & $169(66.5)$ & $573(75.4)$ & 0.007 & $141(67.5)$ & $326(78.2)$ & 0.005 \\
\hline Aspirin & $244(99.2)$ & $711(97.5)$ & 0.185 & $203(99.5)$ & 394 (98.5) & 0.487 \\
\hline Clopidogrel & $28(11.7)$ & $54(7.7)$ & 0.06 & $23(11.5)$ & $34(8.8)$ & 0.24 \\
\hline Prasugrel & $4(1.66)$ & $627(90.0)$ & $<0.001$ & $3(1.5)$ & $339(88)$ & $<0.001$ \\
\hline Ticagrelor & $208(86.7)$ & $18(2.6)$ & $<0.001$ & $174(91.1)$ & $14(3.6)$ & $<0.001$ \\
\hline Statins & $242(97.2)$ & $728(97.5)$ & 1.000 & $198(97.1)$ & $398(98.3)$ & 0.496 \\
\hline ACE-I & $185(85.3)$ & $545(80.3)$ & 0.122 & $150(82.9)$ & $298(80.5)$ & 0.587 \\
\hline ARBs & $26(18.2)$ & $69(12.4)$ & 0.095 & $24(20.5)$ & $49(16.1)$ & 0.349 \\
\hline Beta blockers & $194(85.5)$ & $593(86.7)$ & 0.720 & $161(84.7)$ & $323(86.4)$ & 0.692 \\
\hline
\end{tabular}


Table 3. Ticagrelor compared to prasugrel: 30-day outcomes

\begin{tabular}{|c|c|c|c|}
\hline & Ticagrelor & Prasugrel & $p$ value \\
\hline \multicolumn{4}{|c|}{ (a) STEMI patients - entire cohort } \\
\hline$N$ & 306 & 903 & \\
\hline Re-hospitalization & $52(20.0)$ & $108(13.8)$ & 0.022 \\
\hline RE-MI & $5(1.8)$ & $6(0.7)$ & 0.189 \\
\hline Angina & $7(4.9)$ & $20(3.8)$ & 0.725 \\
\hline CABG & $8(2.9)$ & $11(1.2)$ & 0.113 \\
\hline MACE* & $28(9.3)$ & $43(4.8)$ & 0.006 \\
\hline Mortality & $6(2.0)$ & $8(0.9)$ & 0.224 \\
\hline \multicolumn{4}{|c|}{ (b) STEMI patients PSM cohort } \\
\hline$N$ & 250 & 500 & \\
\hline Re-hospitalization & $41(19.2)$ & $55(12.9)$ & 0.049 \\
\hline RE-MI & $4(1.7)$ & $2(0.4)$ & 0.169 \\
\hline Angina & $7(5.5)$ & $7(2.4)$ & 0.201 \\
\hline CABG & $6(2.6)$ & $4(0.8)$ & 0.121 \\
\hline MACE* & $23(9.3)$ & $20(4.0)$ & 0.006 \\
\hline Mortality & $4(1.6)$ & $4(0.8)$ & 0.528 \\
\hline \multicolumn{4}{|l|}{ (c) NSTEMI-ACS cohort } \\
\hline$N$ & 511 & 223 & \\
\hline Re-hospitalization & $61(13.5)$ & $20(9.9)$ & 0.242 \\
\hline RE-MI & $5(1.1)$ & $3(1.4)$ & 1.000 \\
\hline Angina & $13(5.1)$ & $5(3.7)$ & 0.699 \\
\hline CABG & $2(0.4)$ & $2(0.9)$ & 0.806 \\
\hline MACE* & $20(4.0)$ & $8(3.6)$ & 0.970 \\
\hline Mortality & $1(0.2)$ & $1(0.4)$ & 1.000 \\
\hline
\end{tabular}

RE-MI, recurrent myocardial infarction; CABG, coronary artery bypass grafting. ${ }^{*}$ MACE - major adverse cardiac event including mortality, Ml, stroke, and need for urgent revascularization.

nificant differences between the groups. The rate of inhospital complications was low and comparable in both groups. Outcomes at 30 days were similar in both groups (Table 3). The 1-year mortality rate was comparable between groups $(1.1 \%$ and $2.8 \%$ in the ticagrelor and prasugrel groups, respectively; $p=0.087$; Fig. $3 c$ ). In a Cox model adjusting for age, hypertension, smoking, and $\mathrm{CKD}$, with an interaction term between medication (prasugrel/ticagrelor) and clinical syndrome (STEMI/NSTEACS), the effect of drug choice on 1-year mortality was significantly related to the clinical syndrome ( $p$ for interaction 0.03 , Fig. 4).

\section{Discussion}

The current analysis, from a large multicenter registry in Israel, compares the outcomes of ACS patients undergoing PCI, who were treated with ticagrelor versus prasugrel. Our key findings are (1) prasugrel was more com- monly used in STEMI patients and ticagrelor in NSTEACS patients. (2) Patients treated with ticagrelor were older, with higher rate of CKD, while patients prescribed prasugrel were more commonly smokers. (3) In-hospital complication rate, including rates of stent thrombosis, and bleeding were uncommon and similar between groups. (4) Among STEMI patients, 30-day re-hospitalization rate, 30-day MACE, and 1-year mortality rates were higher in the ticagrelor group compared to the prasugrel group, and among NSTE-ACS patients, outcomes were not affected by drug choice.

The novel P2Y12 inhibitors were incorporated into clinical practice based on their superiority over clopidogrel demonstrated in 2 pivotal trials. In TRITON-TIMI 38 [3], prasugrel was tested in a cohort of high-risk ACS patients with a planned invasive strategy. In NSTE-ACS, the study drug was administered only after the coronary anatomy was delineated and deemed suitable for PCI, while in STEMI, randomization and treatment was permitted at the time of diagnosis. Compared to clopidogrel, prasugrel has shown an absolute risk reduction of $2.2 \%$ in the composite of cardiovascular death, MI, and stroke at 15 months and $50 \%$ relative risk reduction in stent thrombosis. Prasugrel was not better than clopidogrel when tested in ACS patients not planned for invasive strategy [6]. In the PLATO trial [5], ticagrelor was given upon randomization in all ACS patients, with only $61 \%$ eventually undergoing in-hospital PCI. Ticagrelor was superior to clopidogrel with an absolute risk reduction of $1.9 \%$ in the primary composite outcome at 12 months and 16\% relative risk reduction in MI. Remarkably, ticagrelor use was also associated with lower 1-year CV death rate compared to clopidogrel. Based on these observations, the common practice in many centers in Israel is to treat STEMI patients, in whom the vast majority is managed invasively, with prasugrel, while NSTE-ACS patients, who are occasionally managed conservatively or referred to bypass surgery, are treated with ticagrelor.

Observational and registry-based studies comparing prasugrel and ticagrelor in STEMI patients have yielded mixed results. Some studies showed a neutral effect of prasugrel compared to ticagrelor [7-9], whereas others demonstrated superiority of prasugrel driven by lower rates of death and RE-MI and equivalent bleeding rates $[10,11]$. There are few observational data looking specifically at prasugrel versus ticagrelor in NSTE-ACS patients. Notably, PSM data from the RENAMI registry showed superiority of prasugrel over ticagrelor in the subgroup of NSTE-ACS patients, but not in STEMI patients [12]. 
Fig. 3. Kaplan-Meier curves for 1-year death in the following groups: entire STEMI cohort (a), PSM STEMI cohort (b), and NSTE-ACS cohort (c).

Fig. 4. Cox model for 1-year death in the full cohort.

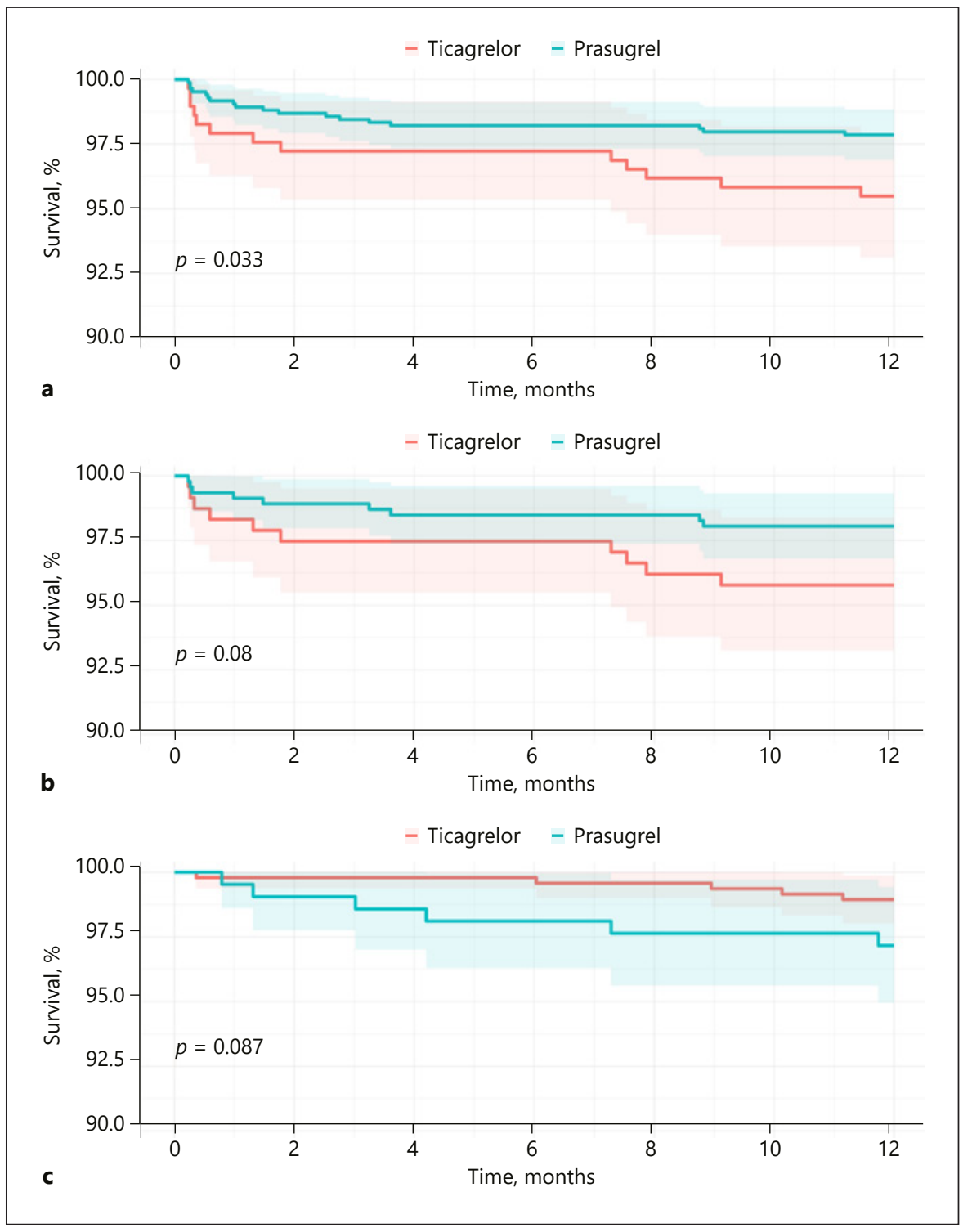

Hazard ratio for 1-year death : Ticagrelor vs. Prasugrel

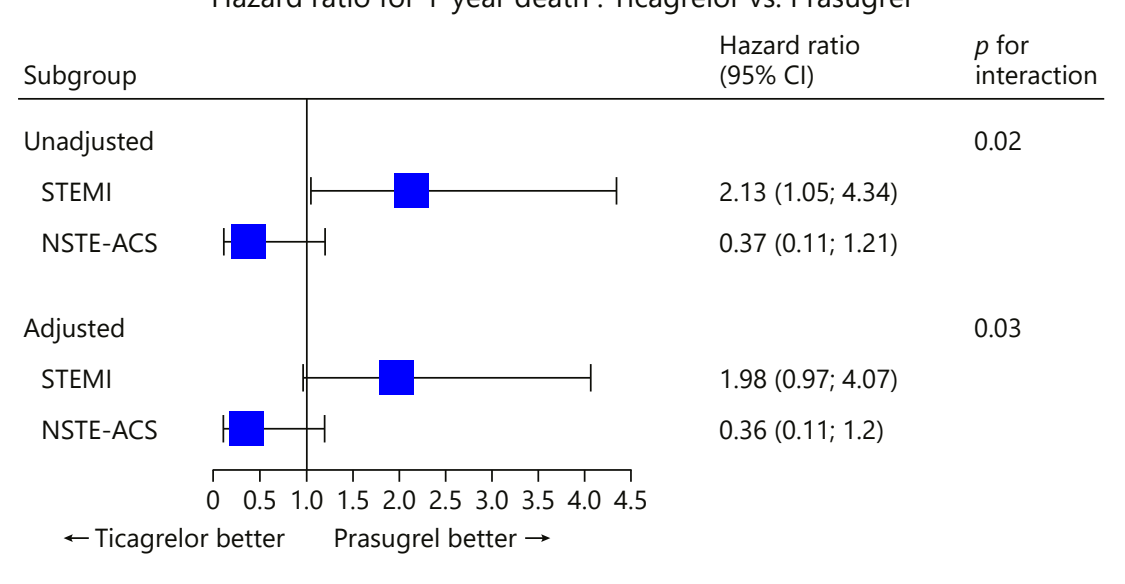


Thus far, 2 randomized clinical trials performed a head-to-head comparison of ticagrelor and prasugrel with conflicting outcomes. The PRAGUE-18 trial compared prasugrel and ticagrelor in STEMI patients undergoing primary PCI [13]. There was no significant difference between the groups in the incidence of the primary outcome (CV death, MI, and stroke at 1 year; $6.6 \%$ and $5.7 \%$ in the prasugrel and ticagrelor groups, respectively). However, this trial was criticized for the high rate of conversion to clopidogrel early after discharge $(34 \%$ in the prasugrel group and $44 \%$ in the ticagrelor group), probably due to lack of reimbursement, undermining the reliability of the study outcomes. The ISAR-REACT- 5 trial compared prasugrel and ticagrelor in patients with ACS (41\% STEMI) who were scheduled to undergo invasive evaluation ( $84 \%$ underwent PCI). Contrary to the study hypothesis, the incidence of the primary endpoint (allcause death, MI, and stroke at 1 year) was significantly lower in the prasugrel arm (6.9\% vs. 9.3\%, $p=0.006)$, driven mainly by lower MI rate (RRR 39\%, ARR 1.8\%). Stent thrombosis rate, as well as bleeding rate, was not significantly different between study arms. The outcomes of ISAR-REACT-5 have raised considerable attention, leading to modification of the ESC NSTEMI 2020 guidelines, which suggested that "prasugrel should be considered in preference to ticagrelor for NSTE-ACS patients who proceed to PCI" as a class IIa recommendation [1]. More recently, prespecified subgroup analyses of the ISAR-REACT-5 were published showing that outcomes both in the STEMI and NSTE-ACS cohorts were consistent with the overall trial result $[14,15]$.

Our study demonstrated superiority of prasugrel over ticagrelor only in STEMI patients with lower incidence of 30-day MACE, 30-day re-hospitalization, and marginally lower 1-year death. Several mechanisms may apply; while both ticagrelor and prasugrel are inhibitors of the adenosine diphosphate receptor P2Y12 on platelets, they are different compounds with different pharmacokinetic and pharmacodynamic properties. Specifically, ticagrelor is a reversible direct platelet inhibitor, while prasugrel is an irreversible pro-drug offering improved protection in case of drug interruption. Prasugrel is taken once daily and ticagrelor twice, potentially leading to improved adherence with prasugrel. Additionally, higher rate of conversion to the less potent $\mathrm{P} 2 \mathrm{Y} 12$ inhibitor clopidogrel after 30 days was observed more commonly in patients taking ticagrelor, possibly related to side effects such as dyspnea and bradycardia. The differences between the agents in terms of pleiotropic and vasoactive effects are somewhat elusive and difficult to delineate. Several stud- ies demonstrated superiority of prasugrel compared with ticagrelor with improved endothelial function, lower platelet reactivity, stronger platelet inhibition, and reduced IL-6 levels [16, 17]. Others are either neutral [18] or favor ticagrelor [19].

In the current analysis, in contradistinction with the findings of the ISAR-REACT-5 trial, the effect of drug choice on 1-year mortality was impacted by the clinical syndrome. The effect of drug choice was neutral in NSTEACS, whereas prasugrel was superior in STEMI. The following explanations may apply: (1) STEMI is a condition associated with a more thrombotic milieu and thus hypothetically requires a more robust platelet inhibition offered by prasugrel to prevent future thrombotic complications. In keeping with this hypothesis, a recently published network meta-analysis shows a lower pooled hazard ratio of stent thrombosis with prasugrel compared to ticagrelor [20]. (2) While only patients undergoing PCI were included in the current analysis, approximately $14 \%$ of the patients in the ISAR-REACT-5 trial were managed conservatively. These patients were not necessarily treated per protocol thus attenuating the effect of the study drugs on outcomes.

The current trial has several limitations. First, given the observational nature of this analysis, we could not rule out that unmeasured factors were associated with patient selection into the drug group. To minimize this bias, we applied multivariable models and PSM analysis. Second, 1 -year outcome variables other than mortality such as MI, stroke, and bleeding rates were not collected in the ACSIS thereby precluding a direct comparison to the outcomes presented in the aforementioned groundbreaking trials. Last, given the limited cohort size and modest treatment effect observed, we cannot overrule the presence of type I or II biases affecting our outcomes. We therefore suggest interpreting our conclusions as hypothesis generating.

In conclusion, the current analysis suggests that prasugrel may be superior to ticagrelor in STEMI patients, but not in NSTE-ACS patients. Further investigation is warranted to affirm our findings and elucidate the mechanisms responsible for this difference.

\section{Statement of Ethics}

Data collection for all ACSIS was approved at each hospital by the local institutional ethics review board. The approval is by the Rabin Medical Center Review Board. Approval Reference: No. 622-17. All patients were informed of the nature and aims of surveys and signed a written informed consent. 


\section{Conflict of Interest Statement}

The authors report no competing interests.

\section{Funding Sources}

The statistical analysis required for this manuscript was performed and funded by the Israeli Center for Cardiovascular Research (ICCR). Otherwise, no funding was used.

\section{Author Contributions}

A.L., R.E., B.M., R.A., and E.K. contributed to conception and design of the research. A.L., R.E., B.M., R.A., M.K., and E.K. contributed to analysis and interpretation of the data. T.O. contributed to statistical analysis. A.L., R.E., B.M., R.A., and E.K. contributed to drafting of the manuscript. R.K., R.B., D.H., and Z.I. contributed to critical revision of the manuscript for important intellectual content.

\section{Data Availability Statement}

All data generated or analyzed during this study are available with the ICCR. Further enquiries can be directed to the corresponding author.

\section{References}

1 Collet JP, Thiele H, Barbato E, Barthelemy O, Bauersachs J, Bhatt DL, et al. 2020 ESC Guidelines for the management of acute coronary syndromes in patients presenting without persistent ST-segment elevation. Eur Heart J. 2021 Apr;42(14):1289-367.

2 O'Gara PT, Kushner FG, Ascheim DD, Casey DE, Chung MK, de Lemos JA, et al. 2013 ACCF/AHA guideline for the management of ST-elevation myocardial infarction. J Am Coll Cardiol. 2013;61:e78-140.

3 Wiviott SD, Braunwald E, McCabe CH, Montalescot G, Ruzyllo W, Gottlieb S, et al. Prasugrel versus clopidogrel in patients with acute coronary syndromes. N Engl J Med. 2007;357 2001-15.

4 Montalescot G, Bolognese L, Dudek D, Goldstein P, Hamm C, Tanguay JF, et al. Pretreatment with prasugrel in non-ST-segment elevation acute coronary syndromes. N Engl J Med. 2013;369:999-1010.

5 Wallentin L, Becker RC, Budaj A, Cannon CP, Emanuelsson $\mathrm{H}$, Held C, et al. Ticagrelor versus clopidogrel in patients with acute coronary syndromes. N Engl J Med. 2009;361: 1045-57.

6 Roe MT, Armstrong PW, Fox KA, White HD, Prabhakaran D, Goodman SG, et al. Prasugrel versus clopidogrel for acute coronary syndromes without revascularization. $\mathrm{N}$ Engl J Med. 2012;367:1297-309.

7 Kim MC, Jeong MH, Sim DS, Hong YJ, Kim $\mathrm{JH}$, Ahn Y, et al. Korea acute myocardial infarction-national institutes of health registry investigators. comparison of clinical outcomes between ticagrelor and prasugrel in patients with ST-segment elevation myocardial infarction results from the korea acute myocardial infarction registry-national institutes of health. Circ J. 2018;82:1866-73.

8 De Luca L, Zeymer U, Claeys MJ, Dörler J, Erne P, Matter CM, et al. Comparison of
P2Y12 receptor inhibitors in patients with ST-elevation myocardial infarction in clinical practice: a propensity score analysis of five contemporary European registries. Eur Heart J. 2020:pvaa002.

9 Venetsanos D, Träff E, Erlinge D, Hagström E, Nilsson J, Desta L, et al. Prasugrel versus ticagrelor in patients with myocardial infarction undergoing percutaneous coronary intervention. Heart. 2021;107(14):1145-51.

10 Krishnamurthy A, Keeble C, Anderson M, Somers K, Burton-Wood N, Harland C, et al. Real-world comparison of clopidogrel, prasugrel and ticagrelor in patients undergoing primary percutaneous coronary intervention. Open Heart. 2019;6:e000951.

11 Olier I, Sirker A, Hildick-Smith DJR, Kinnaird T, Ludman P, de Belder MA, et al. Association of different antiplatelet therapies with mortality after primary percutaneous coronary intervention. Heart. 2018; 104: 1683-90.

12 De Filippo O, Cortese M, D'Ascenzo F, Raposeiras-Roubin S, Abu-Assi E, Kinnaird T, et al. Real-world data of prasugrel versus ticagrelor in acute myocardial infarction: results from the RENAMI registry. Am J Cardiovasc Drugs. 2019;19(4):381-91.

13 Motovska Z, Hlinomaz O, Kala P, Hromadka M, Knot J, Varvarovsky I, et al. One-year outcomes of patients undergoing primary angioplasty for myocardial infarction treated with prasugrel versus ticagrelor. J Am Coll Cardiol. 2018;71:371.

14 Aytekin A, Ndrepepa G, Neumann FJ, Menichelli M, Mayer K, Wöhrle J, et al. Ticagrelor or prasugrel in patients with ST-segmentelevation myocardial infarction undergoing primary percutaneous coronary intervention. Circulation. 2020;142:2329-37.

15 Valina C, Neumann FJ, Menichelli M, Mayer K, Wöhrle J, Bernlochner I, et al. Ticagrelor or prasugrel in patients with non-ST-segment elevation acute coronary syndromes. J Am Coll Cardiol. 2020;76:2436-46.

16 Schnorbus B, Daiber A, Jurk K, Warnke S, Koenig J, Lackner KJ, et al. Effects of clopidogrel versus prasugrel versus ticagrelor on endothelial function, inflammatory parameters, and platelet function in patients with acute coronary syndrome undergoing coronary artery stenting: a randomized, blinded, parallel study. Eur Heart J. 2020;41:3144-52.

17 Mayer K, Bongiovanni D, Karschin V, Sibbing D, Angiolillo DJ, Schunkert H, et al. Ticagrelor or prasugrel for platelet inhibition in acute coronary syndrome patients: the ISARREACT 5 trial. J Am Coll Cardiol. 2020;76: 2569-71.

18 van der Hoeven NW, Janssens GN, Everaars H, Nap A, Lemkes JS, de Waard GA, et al. Platelet inhibition, endothelial function, and clinical outcome in patients presenting with ST-segment-elevation myocardial infarction randomized to ticagrelor versus prasugrel maintenance therapy: long-term follow-up of the REDUCE-MVI trial. J Am Heart Assoc. 2020;9:e014411.

19 Jeong HS, Hong SJ, Cho SA, Kim JH, Cho JY, Lee SH, et al. Comparison of ticagrelor versus prasugrel for inflammation, vascular function, and circulating endothelial progenitor cells in diabetic patients with non-ST-segment elevation acute coronary syndrome requiring coronary stenting: a prospective, randomized, crossover trial. JACC Cardiovasc Interv. 2017;10:1646-58.

20 Navarese EP, Khan SU, Kołodziejczak M, Kubica J, Buccheri S, Cannon CP, et al. Comparative efficacy and safety of oral P2Y12 inhibitors in acute coronary syndrome: network meta-analysis of 52,816 patients from 12 randomized trials. Circulation. 2020;142: $150-60$. 